\title{
Minding the gap in HIV host genetics: opportunities and challenges
}

\author{
Shanelle N. Gingras ${ }^{1,2} \cdot$ David Tang $^{1,2} \cdot$ Jeffrey Tuff ${ }^{1,2} \cdot$ Paul J. McLaren $^{1,2} \mathbb{D}$
}

Received: 16 December 2019 / Accepted: 12 March 2020 / Published online: 14 May 2020

(c) The Author(s) 2020

\begin{abstract}
Genome-wide association studies (GWAS) have been successful in identifying and confirming novel genetic variants that are associated with diverse HIV phenotypes. However, these studies have predominantly focused on European cohorts. HLA molecules have been consistently associated with HIV outcomes, some of which have been found to be population specific, underscoring the need for diversity in GWAS. Recently, there has been a concerted effort to address this gap that leads to health care (disease prevention, diagnosis, treatment) disparities with marginal improvement. As precision medicine becomes more utilized, non-European individuals will be more and more disadvantaged, as the genetic variants identified in genomic research based on European populations may not accurately reflect that of non-European individuals. Leveraging pre-existing, large, multiethnic cohorts, such as the UK Biobank, 23andMe, and the National Institute of Health's All of Us Research Program, can contribute in raising genomic research in non-European populations and ultimately lead to better health outcomes.
\end{abstract}

\section{Genomic studies in HIV: progress to date}

Since the completion of the human genome project, genomewide association studies (GWAS) have identified thousands of reproducible genetic associations with hundreds of complex human traits (Collins et al. 2004; MacArthur et al. 2018). Although chronic diseases, such as type 2 diabetes, have amassed tremendously large sample sizes resulting in $>100$ associated loci (Xue et al. 2018), infectious diseases have only begun to be explored to the same level despite being a major cause of morbidity and mortality globally (Wang et al. 2016). The first GWAS in infectious diseases, performed in people living with HIV (PLWH), identified two independent genetic variants located in human leukocyte antigen (HLA)-B and -C that associated with set point viral load (spVL). This phenotype, defined as mean copies of the HIV RNA genome detected in untreated individuals during

Shanelle N. Gingras

gingrass@myumanitoba.ca; shanelle.gingras@ canada.ca

Paul J. McLaren

Paul.Mclaren@canada.ca

1 JC Wilt Infectious Diseases Research Centre, National HIV and Retrovirology Lab, National Microbiology Laboratories, Public Health Agency of Canada, Winnipeg, Canada

2 Department of Medical Microbiology and Infectious Diseases, University of Manitoba, Winnipeg, Canada the chronic phase of infection (Fellay et al. 2007), correlates both with rate of disease progression to acquired immunodeficiency disease (AIDS) (Mellors et al. 1995) and with transmission potential (Quinn et al. 2000). The observed genetic variants explained $9.6 \%$ and $6.5 \%$ of the variation seen, respectively, and held promise that natural models of HIV control could be informative for design of novel HIV intervention strategies (Fellay 2009; Fellay et al. 2007). Since that first study, technological advances in genomewide platforms have improved the ability to detect and confirm genetic variants identified in GWAS, however, the majority of these studies have been conducted in populations of primarily European descent (Table 1) (MacArthur et al. 2018). The major phenotypes explored in HIV GWAS are: susceptibility to infection, spVL, and disease progression, measured as rate of CD4 T-cell decline (Le Clerc et al. 2009; Herbeck et al. 2010; van Manen et al. 2011). However, studies of these critical phenotypes are much more commonly carried out in European cohorts (Table 1). Furthermore, the majority of studies that do focus on a multiethnic cohort have a greater proportion of European individuals included. With a predominant focus on European individuals, genetic variants driving HIV phenotypes may go undetected.

The largest GWAS done in HIV has been conducted by the International Collaboration for the Genomics of HIV, which has assessed the host genetic contribution to HIV acquisition and spVL (McLaren et al. 2013, 2015). For HIV 


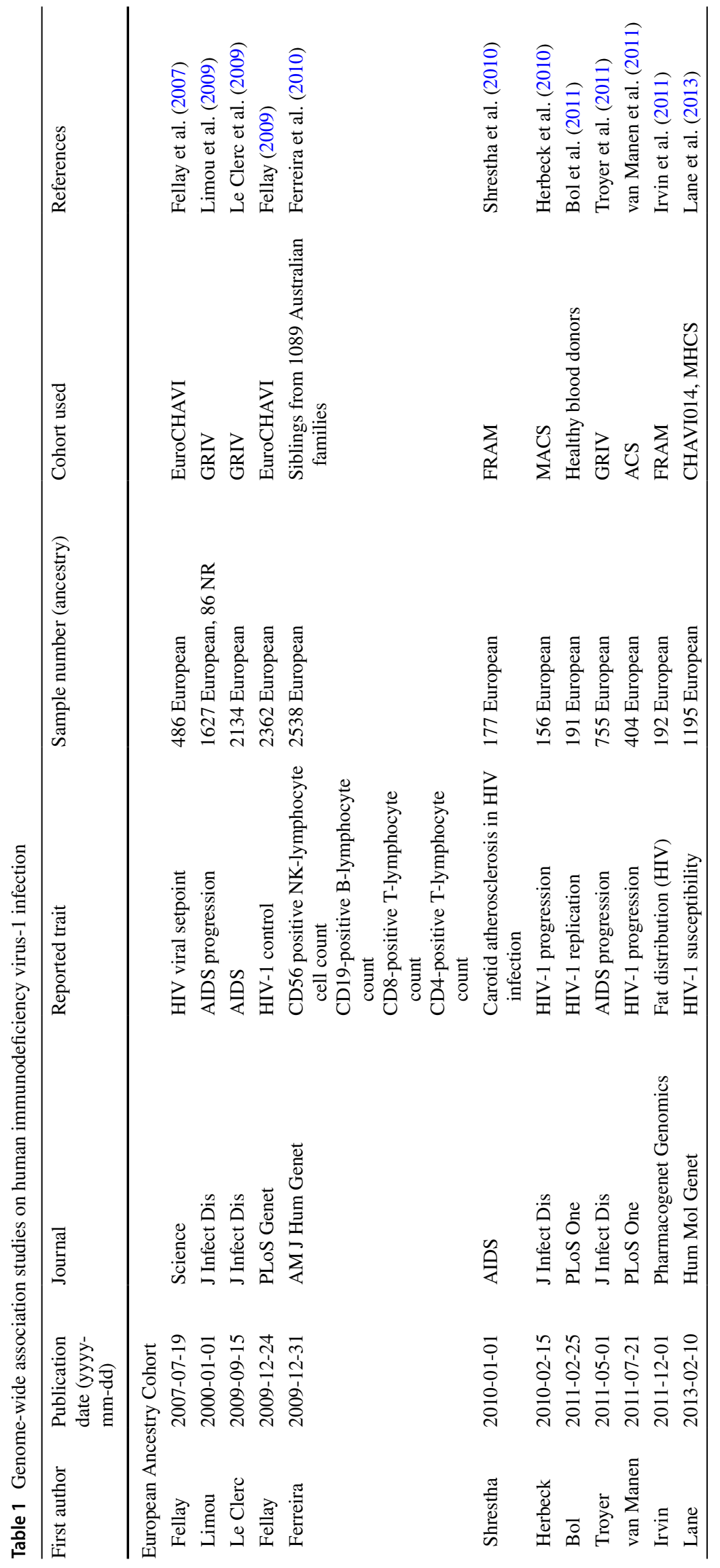




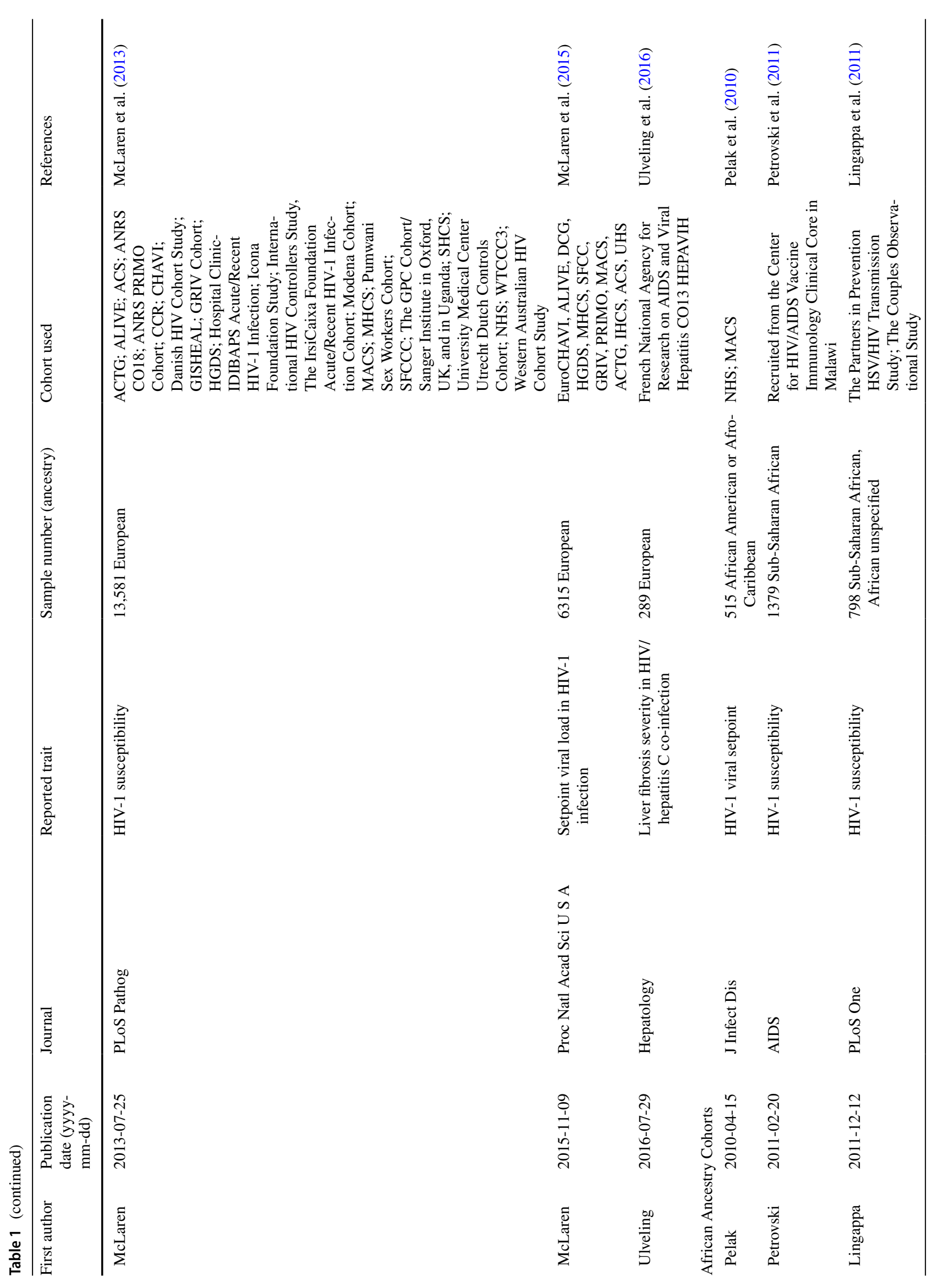




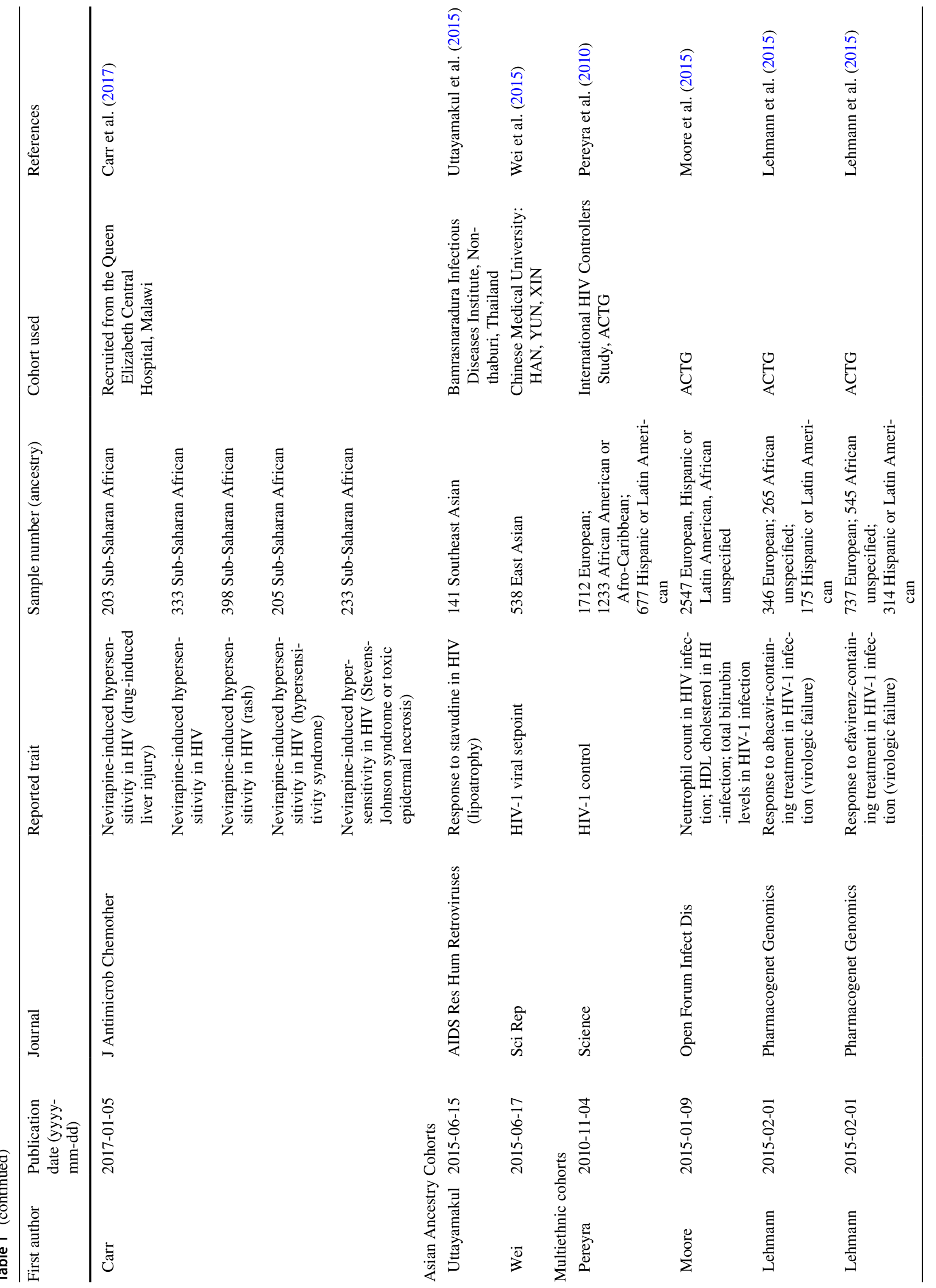




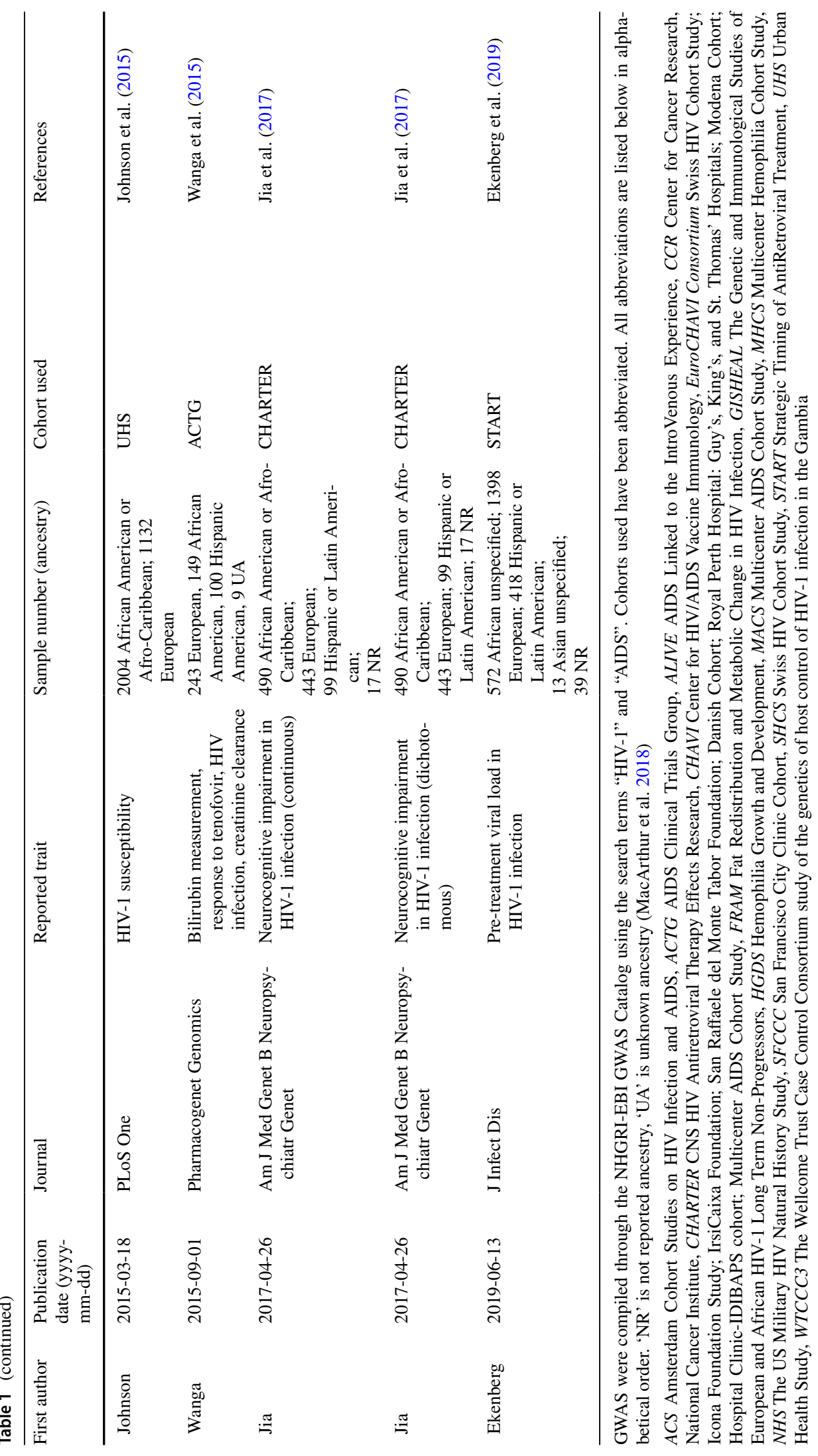


acquisition, although no genome-wide significant variants were identified by comparing $>6000 \mathrm{HIV}$-infected individuals to $>7000$ general population controls, a subsequent analysis did identify significant heritability due to common genetic variants, suggesting larger more diverse samples may uncover novel associations (McLaren et al. 2013; Power et al. 2017). Aggregating data across studies can increase the power to detect associations by addressing the issue of sample size, however, there are many factors to consider for aggregation and subsequent meta-analysis. Data access and exchange can be a difficult balance between protecting the study participants and providing enough information to be useful to the wider scientific community without data breaches, reidentification, or misuse (Zook et al. 2017). Ultimately sharing data positively outweighs the challenges, however, with the stigmatizing nature of an HIV diagnosis, it is imperative that researchers take the proper precautions to protect individuals' privacy. To address these concerns, studies now offer very broad consent forms to enable the future use and exchange of information and there are protocols that must be followed for data exchange and storage to limit the possibility of data breaches (Zook et al. 2017). Metaanalysis historically has required a homogenous population of study, which is problematic for individuals of African ethnicity as diversity is not only higher than the European population, but they are also less represented in genomic research, leading to smaller potential for data aggregation of a homogenous population (Popejoy and Fullerton 2016; Russo 2007). Statistical methods have been developed that not only account for the differences observed between populations (allelic heterogeneity, linkage disequilibrium (LD) patterns, effect sizes), but also takes into account different genotyping platforms (Wang et al. 2012). It is also important to consider overlap of individuals between studies and cohorts when aggregating data together. As we can see in Table 1, GWAS for HIV reuse multiple cohorts and subsets of cohorts for various studies. Aggregation of these data would require accounting for each individual overlap to ensure no bias is introduced. Aggregating the large amount of data available in HIV genomics would be no easy feat, but looking to current databases that have freely available genome and exome data (Genome Aggregation Database (gnomAD), The 1000 Genomes Project Consortium) can serve as an example for data aggregation (Auton et al. 2015; Karczewski et al. 2019). Exome and genome sequencing projects should be undertaken to address the role of rare variation that is not well captured by GWAS, and with a growing number of sequencing projects, it would serve the scientific community well to aggregate these data for future large-scale use.

For spVL, in addition to reaffirming the protective role of HLA-B*57:01, B*27:05, B*14:02 among other alleles, analysis mapping individual amino acid residues in the
HLA-A and B binding grooves showed that strong effects on $\mathrm{spVL}$ were mediated by variable residues (McLaren et al. 2015). As with this largest study, the majority of GWAS of HIV spVL and disease progression have underscored the importance of HLA class I genes in controlling HIV replication and limiting disease progression. HLA class I molecules are responsible for peptide binding specificity and variation within this region has important implications in disease pathogenesis (Pelak et al. 2010; Peprah et al. 2015; Pereyra et al. 2010; Ramsuran et al. 2018). Multiple variants within class I HLA molecules have been found to reduce spVL and be protective for disease progression in HIV, including the population-specific HLA-B*57:01 for Europeans and HLA-B*57:03 for Africans (Fellay 2009; Fellay et al. 2007; McLaren et al. 2013; Pelak et al. 2010; Pereyra et al. 2010). The mechanism of protection attributed to HLA-B alleles has been proposed to be through a more diverse recognition of a broader epitope repertoire and through recognition of more conserved HIV peptides (Arora et al. 2019; Gaiha et al. 2019). However, it has also been proposed that class I HLA alleles may exert non-peptide dependent protective effects. Recently, Ramsuran et al. investigated the impact of HLA-A on control of HIV and its interaction with other HLA molecules (HLA-E and -B) in an ethnically diverse cohort (2018). Similar to HLA-C, HLA-A is differentially expressed based on allotype specificity (Apps et al. 2013; Ramsuran et al. 2018). However, in contrast to HLA-C, the surface expression of HLA-A is 13- to 18-fold higher and is twofold more polymorphic (Ramsuran et al. 2018). The authors observed that HLA-A expression levels positively associated with a higher VL across populations in spite of varying allelic frequencies, an association that was confirmed in a cohort of individuals with known seroconversion dates (Ramsuran et al. 2018). Furthermore, increased HLA-A expression level was strongly associated with elevated mean VL, increased odds of being an HIV non-controller versus controller, and decreased CD4 + T-cell counts (Ramsuran et al. 2018).

The mechanism underlying the impact of HLA-A on HIV progression was further investigated in the context of its relationship to HLA-E. HLA-E is a ligand for the CD94/ NKG2A receptor found on both natural killer (NK) cells and $\mathrm{T}$ cells. When stably bound to a signal peptide produced by the leader sequences of HLA-A, -B, and -C, HLA-E strongly inhibits NK cells activity (Lee et al. 1998; Ramsuran et al. 2018). Interestingly, highly expressed HLA-A alleles significantly correlated with higher expression of HLA-E on the cell surface, independent of the known protective HLA-B alleles, and reduced the control of HIV via NKG2Amediated NK inhibition by interfering with the clearance of HIV-infected cells (Ramsuran et al. 2018). HIV favors the increased expression of HLA-E to decrease the risk of NK cell recognition, yet this immune evasion strategy heightens the risk of restricted CD8 + T-cell recognition (Hansen 
et al. 2016). Recently, researchers have been able to exploit this mechanism by eliciting unconventional CD $8+\mathrm{T}$-cell responses to MHC-E peptide presentation in rhesus macaques (RM) using a rhesus cytomegalovirus (RhCMV) SIVgag vector-based vaccine (Hansen et al. 2013, 2016; Walters et al. 2018). This vaccine broadly enables CD8 + T cells to recognize MHC-E-restricted presentation of highly variable peptides and can be targeted for a pathogen's specific vulnerability through deletions in the RhCMV genome, in the case of HIV, the upregulation of MHC-E on the cell surface (Früh and Picker 2017; Hansen et al. 2013, 2016; Walters et al. 2018). With more than $50 \%$ of the vaccinated RM demonstrating protection to a highly pathogenic SIV strain and infected RM being indiscernible from vaccinated unchallenged RM leading up to 18 months post-infection, targeting of HLA-E upregulation during HIV infection is an attractive strategy moving forward (Früh and Picker 2017; Hansen et al. 2013, 2016; Walters et al. 2018). These data demonstrate the myriad of ways in which HLA may contribute to control and clearance of infection.

Multiple independent genetic associations have also been reported in the CCR5 gene region. The strongest of which, CCR5 $\Delta 32$ confers protection against infection in the homozygous state and slows disease progression in heterozygotes (Dean et al. 1996). Recently, Kulkarni et al. demonstrated that an additional polymorphism in the same region, rs1015164 which also impacts spVL (McLaren et al. 2015), associated with expression of an antisense long noncoding RNA that overlaps CCR5, dubbed CCR5-AS (2019). Expression level of CCR5-AS positively associated with expression of CCR5 and, when depleted, led to a reduction in HIV infection in vitro highlighting an alternative mechanism for CCRR5-related HIV control (Kulkarni et al. 2019).

Host control of HIV has been shown to differ across ethnicities likely due in part to population-specific variants associated with susceptibility to infection and disease progression (Peprah et al. 2015; Pereyra et al. 2010). Frequency and effect size of risk alleles can vary substantially across populations, making it critical to identify associated genetic variation in non-European populations, particularly in Africa where HIV is found in the highest proportion worldwide (Peprah et al. 2015; UNAIDS 2019). Closing this gap is not only important to improve health disparities, but can also improve genetic discovery by uncovering risk alleles not found in European populations. Recently, efforts have been made to improve ethnic diversity in HIV genomic studies. Ekenberg et al. assessed genetic regulators of VL on a subset of the Strategic Timing of Antiretroviral Treatment (START) cohort, that enrolled ART-naïve, ethnically diverse (35 countries from 5 continents), HIV-positive individuals (Ekenberg et al. 2019). Five SNPs met genome-wide significance, all of which were found in the MHC class I region (Ekenberg et al. 2019). The most significant SNP, located upstream of
HLA-B, was identified to tag HLA-B*5701 (Ekenberg et al. 2019). Importantly, the observed variant exhibited high positive predictive values (PPV) in White, Asian, and Hispanic populations, however, in the Black participants PPV was low (Ekenberg et al. 2019). Two more population-specific variants were also confirmed in this study, HLA-B*5701 and HLA-B*5703, with higher frequencies found in European and African populations, respectively (Ekenberg et al. 2019). Two SNPS that have previously been reported to control HIV infection, one located in the HLA complex P5 gene and another in close proximity to the MICA gene, were found to be in moderate LD with the top associated SNP (Ekenberg et al. 2019). The SNP found close to the MICA gene was also found to be statistically significant across all four ethnicities (Ekenberg et al. 2019). These data demonstrate that although there are variants that consistently associate with phenotypes across populations, there are variants that are specific to a population and unique SNPs can tag HLA alleles distinct to a population due to underlying variations in LD structure, reflecting out of Africa migration patterns and the shortening of African LD blocks through increased generational decay (Charles et al. 2014; Ekenberg et al. 2019).

\section{Opportunities for novel discovery using large, multiethnic data sets}

Currently, there are large multiethnic genetic studies that are ongoing to address the disparity in genomic medicine across ancestry groups. One such study, The UK Biobank, consists of 500,000 UK participants enrolled at ages 40-69 from diverse ethnic backgrounds (UK Biobank 2017). All 500,000 participants have been genotyped and electronic health records (general practice, hospital episodes, cancer, death) have been linked to the participants allowing for the joint analysis of genetic, clinical and demographic data on relevant clinical samples (blood, urine, saliva) taken at the time of enrollment for all participants, and at followup appointments for a subset of 20,000 individuals (UK Biobank 2017).

Mentzer et al. used the UK Biobank resource to study the host-pathogen-disease relationship using a Multiplex Serology platform optimized for 20 different pathogens, with three disease endpoints of known (human papillomavirus (HPV)-16 and cervical cancer), potential (Epstein-Barr virus (EBV) and cytomegalovirus (CMV) and multiple sclerosis), and unlikely (coeliac disease) infectious disease association (Mentzer et al. 2019). They observed significant seroprevalence differences among various demographics, including sex, age, lifetime sexual partners, and self-reported ethnicity (hepatitis B virus, CMV) for varying infections, and were able to confirm multiple previously reported 
associations with infectious diseases (Mentzer et al. 2019). Interestingly, the number of lifetime sexual partners was not associated with infectious diseases with well-established sexual routes of transmission (HIV, HBV, HCV, HTLV-1), which appears to be due to the rare occurrences of these particular diseases in this study population (Mentzer et al. 2019). However, when considering HIV and HBV in men who have sex with men, a statistically significant association was identified (Mentzer et al. 2019). The use of large, pre-established, ethnically diverse cohorts is an intriguing option for GWAS moving forward. Samples such as the UK Biobank not only have the power to detect novel variants, but can also help to address the disproportionately low number of non-European cohorts currently seen in genomics, which continues to be exacerbated through the resampling of European cohorts (Popejoy and Fullerton 2016).

Similarly, direct to consumer genomic companies, such as 23 andMe also provide a potential resource for enhanced genetic discovery in multiple populations. 23andMe have currently sold more than 10 million genotyping kits, of which $80 \%$ of customers have consented to participate in ongoing research (23andMe 2019). However, their client base currently mirrors the overall ethnic diversity seen in genomic research, with the majority of their data set being Caucasian and Asian individuals (23andMe 2019; Popejoy and Fullerton 2016). To address this discrepancy, 23 andMe has created multiple initiatives to increase their genetic diversity through the Global Genetics Project, Roots Into the Future, African-American Sequencing Project, and African Genetics Project (23andMe 2019). These initiatives have helped to reach a milestone of 45,000 African Americans genotyped on their platform as of May 2016 (23andMe 2019). Leveraging these data would help to increase the diversity seen in genomic research and ultimately could help to improve precision medicine options in non-Europeans. Currently, 23andMe has studied the impact of genetic variants for common infections (chickenpox, cold sores, mononucleosis, yeast infections, etc.) in a cohort of 200,000 European individuals (Tian et al. 2017). They found 13 genome-wide significant SNPs located within the HLA region, many of which were located in the peptide binding cleft, along with more than 40 SNPs located elsewhere (Tian et al. 2017). Inclusion of multiple ethnic groups in the same type of study will serve to further reduce the ancestry gap in genomic medicine.

\section{Precision medicine in HIV}

With the expansion of available cohorts in terms of sample size, ethnic diversity, demographics, and clinical samples, precision medicine is becoming an increasingly more attainable approach. Precision medicine uses individual differences in genetics, socioeconomic factors, environment, and lifestyle to tailor health care options (disease prevention, diagnosis, treatment) to the individual, instead of the population at large which may not accurately reflect the individual. Genetic precision medicine was implemented in HIV care early on in resource-rich countries, when it was observed that individuals carrying the HLA-B*57:01 allele were at high risk for a hypersensitivity reaction to abacavir, a commonly used antiretroviral drug (Hetherington et al. 2002; Mallal et al. 2002). Pre-screening for this allele was shown to reduce this reaction in predominantly white populations (Mallal et al. 2008).

Adverse events have been observed for other commonly used antiretrovirals, including efavirenz (EFV) which was among the first drug to be co-formulated into single pill regimens for mass rollout globally. Several genetic variants in the drug-metabolizing enzyme CYP2B6 have been associated with high EFV plasma concentration and increased risked of adverse neuropsychiatric effects (Haas et al. 2004; Nyakutira et al. 2008; Rotger et al. 2005). Homozygosity, for one such variant, rs3745274, increases the risk of adverse reaction to EFV by fivefold and, given the population differences in frequency, this risk genotype is much more common in Africans (13.7\%) than Europeans (5.6\%) (Auton et al. 2015). Consistent with this, after the mass rollout of the new three-in-one single pill regimen containing EFV in Zimbabwe, an alarming number of patients stopping the drug due to central nervous system side effects was observed, underscoring the need to understand the distribution of such genotypes across treatment contexts (Nordling 2017). This will only become more necessary as global rollout of ART seeks to put $>80 \%$ of the 37.8 million people living with HIV on ART (Unaids 2020). Genomic pre-screening prior to ART initiation may help to reduce adverse events and could also include profiles for risk of developing longterm metabolic and cardiovascular complications known to be increased in people on ART (Rotger et al. 2010, 2013). The benefit of genomic testing prior to ART initiation will have to be weighed against concerns surrounding patient privacy and data security (Mclaren et al. 2016), however, if the diversity gap in genomics goes unaddressed, precision medicine will primarily benefit European populations. This is further underscored by a recent analysis of the performance of polygenic risk scores in predicting disease in non-European populations which showed an average 50\% reduction in predictive accuracy of these scores in populations of African ancestry (Duncan et al. 2019).

One of the most ambitious initiatives in advancing precision medicine is the National Institute of Health's All of Us Research Program. By 2024, at least 1 million diverse participants living in the United States will have contributed biospecimens, physical measurements, health and lifestyle surveys, socioeconomic data, health records, family medical 
history, and or wearable device data (The All of Us Research Program Investigators 2019). One of the core values of the All of Us Research Program is participant diversity and inclusiveness (The All of Us Research Program Investigators 2019). As of July 2019, more than 175,000 participants contributed biospecimens; of those participants, $51 \%$ are non-white and $80 \%$ meet the All of Us Research Program's definition of being underrepresented in biomedical research (The All of Us Research Program Investigators 2019). Overall, this will be a historic effort in identifying new risk factors (biology, environment and social determinants) to improve diagnosis, treatment and care for diverse populations (The All of Us Research Program Investigators 2019). In addition, valuable population reference panels, such as the African Genome Project will improve ability to interpret results of genome-wide studies (Gurdasani et al. 2015). This approach should be expanded to additional valuable resources such as GTEx to better understand how genetic variability impacts gene expression in multiple cell types in diverse ethnic groups (Lonsdale et al. 2013).

\section{Conclusion}

GWAS have been failing to accurately reflect the genetic diversity seen worldwide due to the extensive research in predominantly European populations. In recent years, there has been a concerted effort to address the gap in genomics between European and non-European cohorts, however, the discrepancy is still pronounced. Underrepresented populations have a greater risk for poorer health outcomes due to subpar prevention, diagnosis, and treatment options for their population. Through inclusivity, genomics has the opportunity to address these health disparities and improve health globally.

\section{Compliance with ethical standards}

Conflict of interest On behalf of all authors, the corresponding author states that there is no conflict of interest.

Open Access This article is licensed under a Creative Commons Attribution 4.0 International License, which permits use, sharing, adaptation, distribution and reproduction in any medium or format, as long as you give appropriate credit to the original author(s) and the source, provide a link to the Creative Commons licence, and indicate if changes were made. The images or other third party material in this article are included in the article's Creative Commons licence, unless indicated otherwise in a credit line to the material. If material is not included in the article's Creative Commons licence and your intended use is not permitted by statutory regulation or exceeds the permitted use, you will need to obtain permission directly from the copyright holder. To view a copy of this licence, visit http://creativecommons.org/licenses/by/4.0/.

\section{References}

23andMe (2019) 23andMe for Scientists I Accelerating Genomics Research. https://research.23andme.com/. Accessed 10 Dec 2019

Apps R et al (2013) Influence of HLA-C expression level on HIV control. Science 340(6128):87-91

Arora J et al (2019) HIV Peptidome-wide association study reveals patient-specific epitope repertoires associated with HIV control. Proc Natl Acad Sci USA 116(3):944-949

Auton A et al (2015) A global reference for human genetic variation. Nature 526(7571):68-74

Bol SM et al (2011) Genome-wide association study identifies single nucleotide polymorphism in DYRK1A associated with replication of HIV-1 in monocyte-derived macrophages. PLoS ONE 6(2):e17190

Carr DF et al (2017) Genome-wide association study of nevirapine hypersensitivity in a sub-saharan african HIV-infected population. J Antimicrob Chemother 72:1152-1162

Charles BA, Daniel S, Charles N (2014) Accounting for linkage disequilibrium in association analysis of diverse populations. Gen Epidemiol 38(3):265-273. https://doi.org/10.1002/gepi.21788

Collins FS, Lander ES, Rogers J, Waterson RH (2004) Finishing the euchromatic sequence of the human genome. Nature 431(7011):931-945

Dean M et al (1996) Genetic restriction of HIV-1 infection and progression to AIDS by a deletion allele of the CKR5 Structural Gene. Hemophilia Growth and Development Study, Multicenter AIDS Cohort Study, Multicenter Hemophilia Cohort Study, San Francisco City Cohort, ALIVE Study. Science 273(5283):1856-1862

Duncan L et al (2019) Analysis of polygenic risk score usage and performance in diverse human populations. Nat Commun 10(1):1-9

Ekenberg C et al (2019) Association between single-nucleotide polymorphisms in HLA alleles and human immunodeficiency virus type 1 viral load in demographically diverse, antiretroviral therapy-naive participants from the strategic timing of antiretroviral treatment trial. J Infect Dis 220(8):1325-1334

Fellay J (2009) Common genetic variation and the control of HIV-1 in humans. PLoS Genet 5(12):e1000791

Fellay J et al (2007) A whole-genome association study of major determinants for host control of HIV-1. Science 317(5840):944-947

Ferreira MAR et al (2010) Quantitative trait loci for CD4:CD8 lymphocyte ratio are associated with risk of type 1 diabetes and HIV-1 immune control. Am J Hum Genet 86(1):88-92

Früh K, Louis P (2017) CD8+ T cell programming by cytomegalovirus vectors: applications in prophylactic and therapeutic vaccination. Curr Opin Immunol 47:52-56

Gaiha GD et al (2019) Structural topology defines protective CD8+ T cell epitopes in the HIV proteome. Science 364(6439):480-484

Gurdasani D et al (2015) The African genome variation project shapes medical genetics in Africa. Nature 517(7534):327-332

Haas DW et al (2004) Pharmacogenetics of efavirenz and central nervous system side effects: an adult AIDS Clinical Trials Group Study. AIDS 18(18):2391-2400

Hansen SG et al (2013) Cytomegalovirus vectors violate CD8+ T cell epitope recognition paradigms. Science 340(6135):1237874

Hansen SG et al (2016) Broadly targeted CD8+ T cell responses restricted by major histocompatibility complex-E HHS public access. Science 351(6274):714-720

Herbeck JT et al (2010) Multistage genomewide association study identifies a locus at $1 \mathrm{q} 41$ associated with rate of HIV-1 disease progression to clinical AIDS. J Infect Dis 201(4):618-626

Hetherington $S$ et al (2002) Genetic variations in HLA-B region and hypersensitivity reactions to abacavir. Lancet 359(9312):1121-1122 
Irvin MR et al (2011) Genes linked to energy metabolism and immunoregulatory mechanisms are associated with subcutaneous adipose tissue distribution in HIV-infected men. Pharmacogen Genom 21(12):798-807

Jia P et al (2017) Genome-Wide Association Study of HIV-associated neurocognitive disorder (HAND): a CHARTER Group Study. Am J Med Genet 174(4):413-426

Johnson EO et al (2015) Novel genetic locus implicated for HIV-1 acquisition with putative regulatory links to HIV replication and infectivity: a Genome-Wide Association Study. PLoS ONE 10(3): 0118149

Karczewski KJ et al (2019) Variation across 141,456 human exomes and genomes reveals the spectrum of loss-of-function intolerance across human protein-coding genes. bioRxiv. https://doi. org/10.1101/531210v3

Kulkarni S et al (2019) CCR5AS LncRNA variation differentially regulates CCR5, influencing HIV disease outcome. Nat Immunol 20(7):824-834

Lane $\mathrm{J}$ et al (2013) A genome-wide association study of resistance to HIV infection in highly exposed uninfected individuals with hemophilia A. Hum Mol Genet 22(9):1903-1910

Le Clerc S et al (2009) Genomewide Association Study of a rapid progression cohort identifies new susceptibility alleles for AIDS (ANRS Genomewide Association Study 03). J Infect Dis 200(8):1194-1201. https://doi.org/10.1086/605892

Lee $\mathrm{Ni}$ et al (1998) HLA-E is a major ligand for the natural killer inhibitory receptor CD94/NKG2A. Proc Natl Acad Sci USA 95(9):5199-5204

Lehmann DS et al (2015) Genome-wide association study of virologic response with Efavirenz-containing or Abacavir-containing regimens in AIDS clinical trials group protocols. Pharmacogenet Genomics 25(2):51-59

Limou S et al (2009) Genomewide Association Study of an AIDSnonprogression cohort emphasizes the role played by HLA genes (ANRS Genomewide Association Study 02). J Infect Dis 199(3):419-426

Lingappa JR et al (2011) Genomewide Association Study for determinants of HIV-1 acquisition and viral set point in HIV-1 serodiscordant couples with quantified virus exposure ed Roberto $\mathrm{F}$ Speck. PLoS ONE 6(12):e28632

Lonsdale J et al (2013) The genotype-tissue expression (GTEx) project. Nat Genet 45(6):580-585

MacArthur JAL et al (2018) NHGRI-EBI GWAS catalog. Nucleic Acids Res 47:1005-1012

Mallal S et al (2002) Association between presence of HLA-B*5701, HLA-DR7, and HLA-DQ3 and hypersensitivity to HIV-1 reversetranscriptase inhibitor abacavir. Lancet 359(9308):727-732

Mallal S et al (2008) HLA-B*5701 screening for hypersensitivity to abacavir. N Engl J Med 358(6):568-579. https://doi.org/10.1056/ NEJMoa0706135

McLaren PJ (2015) Polymorphisms of large effect explain the majority of the host genetic contribution to variation of HIV-1 virus load. Proc Natl Acad Sci USA 112(47):14658-14663

McLaren PJ et al (2013) Association study of common genetic variants and HIV-1 acquisition in 6,300 infected cases and 7,200 controls. PLoS Pathog 9(7):e1003515

Mclaren PJ et al (2016) Privacy-preserving genomic testing in the clinic: a model using HIV treatment. Genet Med 18(8):814-822

Mellors JW et al (1995) Quantitation of HIV-1 RNA in plasma predicts outcome after seroconversion. Ann Intern Med 122(8):573-579

Mentzer AJ et al (2019) Identification of host-pathogen-disease relationships using a scalable multiplex serology platform in UK biobank. medrxiv. https://doi.org/10.1101/19004960

Moore CB et al (2015) Phenome-wide association study relating pretreatment laboratory parameters with human genetic variants in
AIDS Clinical Trials Group Protocols. Open Forum Infect Dis 2(1): 113

Nordling L (2017) How the genomics revolution could finally help Africa. Nature 544(7648):20-22

Nyakutira $\mathrm{C}$ et al (2008) High prevalence of the CYP2B6 516G $\rightarrow \mathrm{T}\left({ }^{*} 6\right)$ variant and effect on the population pharmacokinetics of Efavirenz in HIV/AIDS outpatients in Zimbabwe. Eur J Clin Pharmacol 64(4):357-365

Pelak K et al (2010) Host determinants of HIV-1 control in African Americans. J Infect Dis 201(8):1141-1149

Peprah E et al (2015) Genome-wide association studies in Africans and African Americans: expanding the framework of the genomics of human traits and disease HHS public access. Public Health Genom 18(1):40-51

Pereyra $\mathrm{F}$ et al (2010) The major genetic determinants of HIV-1 control affect HLA class I peptide presentation. Science 330(6010):1551-1557

Petrovski S et al (2011) Common human genetic variants and HIV-1 susceptibility: a genome-wide survey in a homogeneous African population. AIDS 25(4):513-518

Popejoy AB, Fullerton MS (2016) Genomics is failing on diversity. Nature 538(7624):161-164

Power RA et al (2017) A genome-wide polygenic approach to HIV uncovers link to inflammatory bowel disease and identifies potential novel genetic variants. bioRxiv. https://doi. org/10.1101/145383v1

Quinn TC et al (2000) Viral load and heterosexual transmission of human immunodeficiency virus type 1. Rakai Project Study Group. N Engl J Med 342(13):921-929

Ramsuran V et al (2018) Elevated HLA-A expression impairs HIV control through inhibition of NKG2A-expressing cells. Science 359(6371):86-90

Rotger M et al (2005) Influence OfCYP2B6 polymorphism on plasma and intracellular concentrations and toxicity of Efavirenz and Nevirapine in HIV-infected patients. Pharmacogenet Genom 15(1):1-5

Rotger M et al (2010) Impact of single nucleotide polymorphisms and of clinical risk factors on new-onset diabetes mellitus in HIV-infected individuals. Clin Infect Dis 51(9):1090-1098

Rotger M et al (2013) Contribution of genetic background, traditional risk factors, and HIV-related factors to coronary artery disease events in HIV-positive persons. Clin Infect Dis 57(1):112-121

Russo MW (2007) How to review a meta-analysis. Gastroenterol Hepatol 3(8):637-642

Shrestha $S$ et al (2010) A genome-wide association study of carotid atherosclerosis in HIV-infected men. AIDS 24(4):583-592

The All of Us Research Program Investigators (2019) The 'All of Us' research program. N Engl J Med 381(7):668-676. https://doi. org/10.1056/NEJMsr1809937

Tian C et al (2017) Genome-wide association and HLA region finemapping studies identify susceptibility loci for multiple common infections. Nature Communications 8(1):599

Troyer JL et al (2011) Genome-wide association study implicates PARD3B-based AIDS restriction. J Infect Dis 203(10):1491-1502

UK Biobank. 2017. UK Biobank Showcase User Guide UK Biobank showcase user guide: getting started. https://www.ukbiobank. ac.uk. Accessed 15 Dec 2019).

Ulveling D et al (2016) A new 3p25 locus is associated with liver fibrosis progression in human immunodeficiency virus/Hepatitis C virus-coinfected patients. Hepatology 64(5):1462-1472

UNAIDS (2019) Global HIV \& AIDS Statistics-2019 Fact Sheet I UNAIDS. https://www.unaids.org/en/resources/fact-sheet. Accessed 9 Sept 2019

Unaids (2020) 90-90-90: an ambitious treatment target to help end the aids epidemic 
Uttayamakul S et al (2015) Genome-Wide Association Study of HIVrelated lipoatrophy in Thai patients: Association of a DLGAP1 polymorphism with fat loss. AIDS Res Hum Retroviruses 31(8):792-796

van Manen D et al (2011) Genome-wide association scan in HIV-1-infected individuals identifying variants influencing disease course. PLoS ONE 6(7):e22208

Walters LC et al (2018) Pathogen-derived HLA-E bound epitopes reveal broad primary anchor pocket tolerability and conformationally malleable peptide binding. Nat Commun 9(1):1-13. https ://doi.org/10.1038/s41467-018-05459-z

Wang X et al (2012) A statistical method for region-based meta-analysis of genome-wide association studies in genetically diverse populations. Eur J Hum Genet 20(4):469-475

Wang H et al (2016) Global, regional, and national life expectancy, all-cause mortality, and cause-specific mortality for 249 causes of death, 1980-2015: a systematic analysis for the global burden of disease study 2015. The Lancet 388(10053):1459-1544
Wanga V et al (2015) Genomewide Association Study of Tenofovir pharmacokinetics and creatinine clearance in AIDS clinical trials group protocol A5202. Pharmacogenet Genom 25(9):450-461

Wei Z et al (2015) Genome-wide association studies of HIV-1 host control in ethnically diverse Chinese populations. Sci Rep 5:10879

Xue A et al (2018) Genome-wide association analyses identify 143 risk variants and putative regulatory mechanisms for type 2 diabetes. Nat Commun 9(1):1-14

Zook M et al (2017) Ten simple rules for responsible big data research ed Fran Lewitter. PLoS Comput Biol 13(3):e1005399. https://doi. org/10.1371/journal.pcbi.1005399

Publisher's Note Springer Nature remains neutral with regard to jurisdictional claims in published maps and institutional affiliations. 